\title{
OPTIMAL LIQUIDATION OF A LARGE BLOCK OF STOCK WITH REGIME SWITCHING
}

\author{
DONG-HOON SHIN
}

\begin{abstract}
This work is concerned with an optimal selling rule for a large position of stock in a market. Selling a large block of stock in a short period typically depresses the market, which would result in a poor filling price. In addition, the large selling intensity makes the regime more likely to be poor state in the market. In this paper, regime switching and depressing terms associated with selling intensity are considered on a set of geometric Brownian models to capture movements of underlying asset. We also consider the liquidation strategy to sell much smaller number of shares in a long period. The goal is to maximize the overall return under state constraints. The corresponding value function with the selling strategy is shown to be a unique viscosity solution to the associated HJB equations. Optimal liquidation rules are characterized by a finite difference method. A numerical example is given to illustrate the result.
\end{abstract}

\section{Introduction}

For financial portfolio management and successful trading of assets, stock liquidation decision is a crucial component. Øksendal [5] formulated the optimal selling decision making as an optimal stopping problem and derived a closed-form solution. Guo and Zhang [3] extended the results as two states are incorporating possible regime switching represented by a two-state Markov chain. Another approach is to consider liquidation decisions by two threshold levels, a target price and a stop-loss limit. The objective is to maximize an expected return from selecting these threshold levels. Zhang [8] obtained optimal threshold levels by solving a set of two boundary value problems. Pemy and Zhang [6] studied a selling rule in which the liquidation is constrained in a specific time period.

The selling of the aforementioned studies is supposed to be done all at once. This is feasible when one deals a relatively small amount of shares of a stock, so

Received November 30, 2009; Revised May 5, 2010.

2010 Mathematics Subject Classification. 91G80.

Key words and phrases. regime switching, optimal selling rule, optimal control, HJB equation, viscosity solution.

This work was supported by Seoul R\&BD Program(10551).

(C)2011 The Korean Mathematical Society 
the selling behavior doesn't affect to the stock price. However, if one sells a large block of shares of the stock in a short period time, the market will be depressed by resulting in poor filling prices. A typical strategy to sell a large amount of a stock is to sell much smaller number of shares over a relatively longer period. Pemy et al. [7] considered large block stock liquidation in the situation. In [7], the stock price is governed by $d X(t)=X(t)(\mu d t+\sigma d w(t))-L(X(t), u(t)) d t$, in which the term $L(X(t), u(t)) d t$ reflects the influence that is related to the rate of selling $u(t)$. In [6], a regime-switching geometric Brownian motion (GBM) model was used instead of the price depressing term $L(X(t), u(t)) d t$.

In this paper, we consider a revised regime-switching GBM model that also involves the price depressing term $L(X(t), u(t)) d t$. We treat the selling rule problem using a fluid model on where the liquidation is thought as the rate of selling over time. Our goal is maximizing the overall return where the underlying stock price model has specific constraints which have a depressing term on geometric Brownian motions with regime switching between two Markov states in which the jump rate depends on the selling intensity. A large selling intensity affects negatively to the market, i.e., the market state changes from higher return mode to lower one or stays at the lower return mode longer. To get the maximum return as our objectives, we use viscosity solution approach to characteristics the dynamics governing the value function with boundary conditions. We use a pair of variables, the stock price and the size of the stock yet sold at time $t$. In the numerical experiments, we will see the state-dependent "threshold" curve to separate two regions, the selling region and the no-selling region. We use a finite difference method for the associated Hamilton-JacobiBellman (HJB) equations and also study its convergence.

This paper is organized as follows. In Section 2, the problem is formulated as a stochastic control problem. Through Section 3, we demonstrate the properties of the optimal value function and uniqueness of the viscosity solution to the system of HJB equations. We establish the associated boundary conditions and optimal selling rate. A finite difference method of the associated HJB equations is introduced in Section 4. Section 5 shows numerical examples containing the method to find the optimal selling rule. In Section 6 , we conclude the paper.

\section{Problem formulation}

Let $\mathcal{M}=\{1,2\}$ and $\alpha(t) \in \mathcal{M}$ be a two-state continuous-time observable Markov chain. Let $X(t)$ denote the stock price, $Z(t)$ be the number of shares of a stock yet sold, $u(t)$ be the rate of selling. Let $\Omega=(0, \infty) \times(0, N)$ be the state space with a finite $N$ being the total number of shares of the stock to be sold. Let $\bar{\Omega}=(0, \infty) \times[0, N]$. Consider the case that the maximum selling rate is 1 . Let $\Gamma=[0,1]$ denote the control set. Assume that the stock price $X(t)$ and $Z(t)$ follows the dynamics

(1) $\left\{\begin{array}{l}d X(t)=X(t)[\mu(\alpha(t)) d t+\sigma(\alpha(t)) d w(t)]-L(X(t), u(t)) d t, X(0)=x, \\ d Z(t)=-u(t) d t, \quad Z(0)=z, u(t) \in \Gamma\end{array}\right.$ 
where $\mu(i)$ and $\sigma(i)$ are known constants, for each state $i \in \mathcal{M}$. The large firm effect $L(X(t), u(t))$ can be various assumption for the market environment in terms of the firm. It can be zero if the firm is enough small, or exponentially increasing function in terms of $u(t)$ if other firms follow the behavior of the large firm, so that if the firm sells the stock, other firms join in the selling as well. However, in this paper, we will suppose that the effect is proportional to the sales $u(t)$ at a rate $c>0$, i.e., $L(X(t), u(t))=c u(t)$. The generator of the Markov chain $\alpha(t)$ is given by $Q(u)=\left(\begin{array}{cc}-\lambda_{1}(u) & \lambda_{1}(u) \\ \lambda_{2}(u) & -\lambda_{2}(u)\end{array}\right)$, where $\lambda_{1}(\cdot)>0$ and $\lambda_{2}(\cdot)>0$. We assume that the jump rates $\lambda_{1}(\cdot)$ and $\lambda_{2}(\cdot)$ have the form

$$
\left\{\begin{array}{l}
\lambda_{1}(u)=a_{1} u+b_{1}, \quad a_{1}>0, b_{1}>0, \\
\lambda_{2}(u)=-a_{2} u+b_{2}, \quad 1 \geq a_{2}>0, b_{2}>0, b_{2}-a_{2}>0 .
\end{array}\right.
$$

Let $\rho>0$ be a discount rate (or an impatient factor).

Definition 2.1. We call that a control $u(\cdot)$ is admissible with respect to the initial values $(x, z, i) \in \bar{\Omega} \times \mathcal{M}$, if

i) $u(\cdot)$ is $\mathcal{F}_{t}=\sigma\{X(s), \alpha(s): s \leq t\}$ adapted;

ii) $u(t) \in \Gamma$ for all $t \geq 0$;

iii) the corresponding state process $(X(t), Z(t)) \in \Omega$ for all $t \geq 0$.

$\mathcal{A}=\mathcal{A}(x, z, i)$ denotes the set of all admissible controls with respect to $(x, z, i)$. We define

$$
J(x, z, i, u(\cdot))=E\left[\int_{0}^{\infty} e^{-\rho t} g(X(t), u(t)) d t \mid X(0)=x, Z(0)=z, \alpha(0)=i\right],
$$

where $g(x, u)$ is the running payoff function. The problem is to choose the rate of selling $u(\cdot) \in \mathcal{A}$ so as to maximize the expected reward $J(x, z, i, u(\cdot))$. The value function for the optimal reward is

$$
v(x, z, i)=\sup _{u(\cdot) \in \mathcal{A}} J(x, z, i, u(\cdot)) .
$$

The system of associated HJB equations is given by

$$
\begin{gathered}
\rho v(x, z, i)=\max _{u \in \Gamma}\left[(\mu(i) x-c u) \frac{\partial v(x, z, i)}{\partial x}-u \frac{\partial v(x, z, i)}{\partial z}+\frac{1}{2} \sigma^{2}(i) x^{2} \frac{\partial^{2} v(x, z, i)}{\partial x^{2}}\right. \\
+g(x, u)+Q(u) v(x, z, \cdot)(i)], \quad 0 \leq z \leq N, i \in\{1,2\},
\end{gathered}
$$

where

$$
\left\{\begin{array}{l}
Q(u) v(x, z, \cdot)(1)=\lambda_{1}(u)(v(x, z, 2)-v(x, z, 1)), \\
Q(u) v(x, z, \cdot)(2)=\lambda_{2}(u)(v(x, z, 1)-v(x, z, 2)) .
\end{array}\right.
$$

Definition 2.2. $v(z, x, i)$ is a viscosity solution of $(3)$ if

i) for each $i_{0} \in \mathcal{M}, v\left(z, x, i_{0}\right)$ is a viscosity supersolution of (3) on $\bar{\Omega}$, i.e.,

$$
\begin{aligned}
& \rho v\left(x_{0}, z_{0}, i_{0}\right)-\max _{u \in \Gamma}\left[\left(\mu\left(i_{0}\right) x_{0}-c u\right) \frac{\partial \phi\left(x_{0}, z_{0}\right)}{\partial x}-u \frac{\partial v\left(x_{0}, z_{0}\right)}{\partial z}\right. \\
& \left.+\frac{1}{2} \sigma^{2}\left(i_{0}\right) x_{0}^{2} \frac{\partial^{2} \phi\left(x_{0}, z_{0}\right)}{\partial x^{2}}+g\left(x_{0}, u\right)+Q(u) v\left(x_{0}, z_{0}, \cdot\right)\left(i_{0}\right)\right] \geq 0,
\end{aligned}
$$


where $\phi(\cdot, \cdot) \in C^{2,1}(\bar{\Omega})$ such that $v\left(x, z, i_{0}\right)-\phi(x, z)$ has a local minimum at $\left(x_{0}, z_{0}\right) \in \bar{\Omega}$.

ii) for each $i_{0} \in \mathcal{M}, v\left(z, x, i_{0}\right)$ is a viscosity sbsolution of (3) on $\bar{\Omega}$, i.e.,

$$
\begin{aligned}
& \rho v\left(x_{0}, z_{0}, i_{0}\right)-\max _{u \in \Gamma}\left[\left(\mu\left(i_{0}\right) x_{0}-c u\right) \frac{\partial \phi\left(x_{0}, z_{0}\right)}{\partial x}-u \frac{\partial v\left(x_{0}, z_{0}\right)}{\partial z}\right. \\
& \left.+\frac{1}{2} \sigma^{2}\left(i_{0}\right) x_{0}^{2} \frac{\partial^{2} \phi\left(x_{0}, z_{0}\right)}{\partial x^{2}}+g\left(x_{0}, u\right)+Q(u) v\left(x_{0}, z_{0}, \cdot\right)\left(i_{0}\right)\right] \leq 0,
\end{aligned}
$$

where $\phi(\cdot, \cdot) \in C^{2,1}(\bar{\Omega})$ such that $v\left(x, z, i_{0}\right)-\phi(x, z)$ has a local maximum at $\left(x_{0}, z_{0}\right) \in \bar{\Omega}$.

\section{Properties of value function}

We demonstrate properties of the value function in this section. First of all, we show that the value function $v(x, z, i)$ is Lipschitz continuous in $(x, z)$ for each $i$. Then we show that the value function is actually the unique solution of the HJB equation (3) in the sense of viscosity solution. Second, we provide boundary conditions for the optimal selling policy. As the last part of this section, we discuss the optimal selling rule from the value function.

Then next two assumptions are needed.

i) $\rho>\mu(1)>\mu(2)$.

ii) $g(x, u)=g_{0} u$ such that $g_{0}$ is twice continuously differentiable and $x g_{0}^{\prime}(x), x^{2} g_{0}^{\prime \prime}(x)$ are bounded.

Remark 3.1. The representative function $g_{0}(x)$ for this paper is $g_{0}(x)=\min \{x$, $M\}$ for a large enough $M$ since a stock price is finite in practice. However, to satisfy the condition of Definition 2.2 , we can choose $g_{0}(x)$ as a convolution of $\min \{x, M\}$ with the kernel

$$
q_{\eta_{0}}(x)=\frac{1}{\sqrt{2 \pi} \eta_{0}} \exp \left(-\frac{x^{2}}{2 \eta_{0}^{2}}\right)
$$

and send $\eta_{0}$ to zero. We have $g_{0}(x)$ as an approximation of $\min \{x, M\}$.

\subsection{Properties of value function}

Theorem 3.2. The following assertions hold.

(a) For each $i$ and $x, v(x, z, i)$ is non-decreasing in $z$.

(b) For each $i, v(x, z, i)$ is Lipschitz continuous in $(x, z)$. More precisely, there exist constants $K_{1}$ and $K_{2}$ such that

$$
\begin{aligned}
& \quad\left|v\left(x_{1}, z_{1}, i\right)-v\left(x_{2}, z_{2}, i\right)\right| \leq K_{1}\left|x_{1}-x_{2}\right|+K_{2}\left|z_{1}-z_{2}\right| \\
& \text { for }\left(x_{1}, z_{1}\right) \text { and }\left(x_{2}, z_{2}\right) \text { in } \bar{\Omega} \text {. }
\end{aligned}
$$

Proof. To verify (a), let $i \in \mathcal{M}$, note that for $0 \leq z_{1} \leq z_{2} \leq N, \mathcal{A}\left(x, z_{1}, i\right) \subset$ $\mathcal{A}\left(x, z_{2}, i\right)$. Given $u(\cdot) \in \mathcal{A}\left(x, z_{1}, i\right)$, then $u(\cdot) \in \mathcal{A}\left(x, z_{2}, i\right)$, and

$$
v\left(x, z_{2}, i\right) \geq J\left(x, z_{2}, i, u(\cdot)\right)=J\left(x, z_{1}, i, u(\cdot)\right) .
$$

This implies $v\left(x, z_{2}, i\right) \geq v\left(x, z_{1}, i\right)$ because $u(\cdot)$ is arbitrary. 
Next, we prove (b). We know that

$$
\begin{aligned}
X(t) & =x+\int_{0}^{t}(\mu(\alpha(s)) X(s)-c u(s)) d s+\int_{0}^{t} \sigma(\alpha(s)) X(s) d w(s) \\
& \leq x+\int_{0}^{t}(\mu(\alpha(s)) X(s)) d s+\int_{0}^{t} \sigma(\alpha(s)) X(s) d w(s) \\
& =x \exp \left(\int_{0}^{t}\left(\mu(\alpha(s)) X(s)-\sigma(\alpha(s))^{2} / 2\right) d s+\int_{0}^{t} \sigma(\alpha(s)) X(s) d w(s)\right) .
\end{aligned}
$$

Note that for any $x_{1}>0$ and $x_{2}>0, \mathcal{A}\left(x_{1}, z, i\right)=\mathcal{A}\left(x_{2}, z, i\right)$ for $0 \leq z \leq N$. Let

$$
H(t)=\int_{0}^{t}\left(\mu(\alpha(s))-\sigma(\alpha(s))^{2} / 2\right) d s+\int_{0}^{t} \sigma(\alpha(s)) d w(s) .
$$

Then, we estimate $E X(t) \leq x E e^{H(t)}$. By Dynkin's formula, we have

$$
E e^{H(t)}=1+\int_{0}^{t} E\left[e^{H(s)} \mu(\alpha(s))\right] d s \leq 1+\mu(1) \int_{0}^{t} E\left[e^{H(s)}\right] d s .
$$

Using Gronwall's inequality, we have

$$
E e^{H(t)} \leq e^{\mu(1) t}
$$

It implies that

$$
E X(t) \leq x E e^{H(t)} \leq x e^{\mu(1) t}
$$

Thus, we can show

$$
E e^{-\rho \tau} X(\tau) \leq x \text { for any stopping time } \tau \text {. }
$$

Given $x_{1}>0$ and $x_{2}>0$, for each $u(\cdot) \in \mathcal{A}\left(x_{1}, z, i\right)=\mathcal{A}\left(x_{2}, z, i\right)$, we have

$\left|J\left(x_{1}, z, i, u(\cdot)\right)-J\left(x_{2}, z, i, u(\cdot)\right)\right| \leq\left|x_{1}-x_{2}\right| E \int_{0}^{\infty} e^{-(\rho-\mu(1)) t} d t=K_{1}\left|x_{1}-x_{2}\right|$.

Since $u(\cdot)$ is arbitrary, we have

$$
\begin{aligned}
\left|v\left(x_{1}, z, i\right)-v\left(x_{2}, z, i\right)\right| & \leq \sup _{u(\cdot) \in \mathcal{A}\left(x_{1}, z, i\right)}\left|J\left(x_{1}, z, i, u(\cdot)\right)-J\left(x_{2}, z, i, u(\cdot)\right)\right| \\
& \leq \frac{\left|x_{1}-x_{2}\right|}{\rho-\mu(1)} .
\end{aligned}
$$

Therefore, $v(x, z, i)$ is Lipschitz in $x$. We only remained to show that $v(x, z, i)$ is Lipschitz in $z$. From (a) and (7), we can derive that for $0 \leq z_{1}<z_{2} \leq N$,

$$
v\left(x, z_{1}, i\right) \geq v\left(x, z_{2}, i\right)-K_{2}\left|z_{1}-z_{2}\right| .
$$

Let $u(\cdot) \in \mathcal{A}\left(x, z_{2}, i\right)$ such that $z_{2}-\int_{0}^{\infty} u_{0}(s) d s=0$ and

$$
v\left(x, z_{2}, i\right) \leq J\left(x, z_{2}, i, u_{0}(\cdot)\right)+\left|z_{1}-z_{2}\right| .
$$

Let $\tau=\inf \left\{t>0: \int_{0}^{\infty} u_{0}(s) d s=z_{2}-z_{1}\right\}$. Obviously,

$$
\int_{0}^{\tau} u_{0}(s) d s=z_{2}-z_{1} .
$$


Define

$$
\tilde{u}(\cdot)= \begin{cases}0 & \text { if } 0 \leq t<\tau \\ u_{0}(t) & \text { if } t \geq \tau .\end{cases}
$$

Then, $\tilde{u}(\cdot) \in \mathcal{A}\left(x, z_{1}, i\right)$. Moreover

$$
\begin{aligned}
\left|J\left(x, z_{2}, i, u_{0}(\cdot)\right)-J\left(x, z_{1}, i, \tilde{u}_{0}(\cdot)\right)\right| & \leq \int_{0}^{\infty} e^{-\rho t} g_{0}(X(t))\left|u_{0}(t)-\tilde{u}(t)\right| d t \\
& =\int_{0}^{\tau} e^{-\rho t} g_{0}(X(t)) u_{0}(t) d t .
\end{aligned}
$$

Next, we show

$$
\int_{0}^{\tau} e^{-\rho t} g_{0}(X(t)) u_{0}(t) d t \leq K\left|z_{1}-z_{2}\right| .
$$

Using integration by parts, we have

$$
\begin{aligned}
& E \int_{0}^{\tau} e^{-\rho t} g_{0}(X(t)) u_{0}(t) d t \\
= & E\left[e^{-\rho \tau} g_{0}(X(\tau))\right] \int_{0}^{\tau} u_{0}(t) d t+E \int_{0}^{\tau} e^{-\rho t}\left(\int_{0}^{\tau} u_{0}(s) d s\right)\left(g_{0}(X(t))\right. \\
& \left.-\mu(\alpha(t)) X(t) g_{0}^{\prime}(X(t))-\frac{\sigma(\alpha(t))^{2}}{2} X^{2}(t) g_{0}^{\prime \prime}(X(t))\right) d t .
\end{aligned}
$$

From (7) and (8), we obtain

$$
E\left[e^{-\rho \tau} g_{0}(X(\tau))\right] \int_{0}^{\tau} u_{0}(s) d s \leq K\left(z_{2}-z_{1}\right)=K\left|z_{1}-z_{2}\right| .
$$

Similarly, using (6) and (8), we conclude

$$
E \int_{0}^{\tau} e^{-\rho t}\left(\int_{0}^{t} u_{0}(s) d s\right) g_{0}(X(t)) d t \leq K\left|z_{1}-z_{2}\right| .
$$

Therefore,

$$
\left|J\left(x, z_{2}, i, u_{0}(\cdot)\right)-J\left(x, z_{1}, i, u_{0}(\cdot)\right)\right| \leq K\left|z_{1}-z_{2}\right| .
$$

It implies that

$$
\begin{aligned}
v\left(x, z_{1}, i\right) & \geq J\left(x, z_{1}, i, \tilde{u}_{0}(\cdot)\right) \geq J\left(x, z_{2}, i, u_{0}(\cdot)\right)-K\left|z_{1}-z_{2}\right| \\
& \geq v\left(x, z_{2}, i\right)-K\left|z_{1}-z_{2}\right| .
\end{aligned}
$$

This completes the proof.

Theorem 3.3. The value function $v(x, z, i)$ is a unique viscosity solution on $\bar{\Omega} \times \mathcal{M}$.

Proof. For any stopping time $\tau$, the dynamic programming principle implies that for each $i$,

(9) $\quad v(x, z, i)=\max _{u(\cdot) \in \mathcal{A}} E\left[\int_{0}^{\tau} e^{-\rho t} X(t) u(t) d t+e^{-\rho \tau} v(X(\tau), Z(\tau), \alpha(\tau))\right]$. 
Let $i_{0} \in \mathcal{M}$, given $(X(0), Z(0))=\left(x_{0}, z_{0}\right) \in \bar{\Omega}$, let $\phi(x, z) \in C^{2,1}(\bar{\Omega})$ such that $v\left(x, z, i_{0}\right)-\phi(x, z)$ has a local minimum in $N\left(x_{0}, z_{0}\right)$, a neighborhood of $\left(x_{0}, z_{0}\right)$. Without loss of generality, we assume that $v\left(x_{0}, z_{0}, i_{0}\right)-\phi\left(x_{0}, z_{0}\right)=0$. Let $\tau$ be the first jump time of $\alpha(\cdot)$. Let $\tau_{0} \in(0, \tau]$ be a stopping time such that for $0 \leq t \leq \tau_{0},(X(t), Z(t)) \in N\left(x_{0}, z_{0}\right)$. Consider the control $u(t)=u \in \Gamma$ for $t \in\left[0, \tau_{0}\right]$, where $u$ is a constant such that $u(\cdot) \in \mathcal{A}\left(x_{0}, z_{0}, i_{0}\right)$. Moreover, for $t \in\left[0, \tau_{0}\right], v(X(t), Z(t), i)-\phi(X(t), Z(t)) \geq 0$. Given $0<\theta \leq \tau_{0}$, using (9), we have

$$
v\left(x_{0}, z_{0}, i_{0}\right) \geq E\left[\int_{0}^{\theta} e^{-\rho s} X(s) u(s) d s+E e^{-\rho \theta} v(X(\theta), Z(\theta), \alpha(\theta))\right] .
$$

Define

$$
\psi(x, z, i)= \begin{cases}\phi(x, z)+v\left(x_{0}, z_{0}, i\right)-\phi\left(x_{0}, z_{0}\right), & \text { if } i=i_{0} \\ v(x, z, i), & \text { if } i \neq i_{0}\end{cases}
$$

Using Dynkin's formula, we derive

$$
\begin{aligned}
& E e^{-\rho \theta} \psi\left(X(\theta), Z(\theta), i_{0}\right)-\phi\left(x_{0}, z_{0}\right) \\
= & E \int_{0}^{\theta}\left[\left(\mu\left(i_{0}\right) X(s)-c u(s)\right) \frac{\partial \phi(X(s), Z(s))}{\partial x}\right. \\
& -u(s) \frac{\partial \phi(X(s), Z(s))}{\partial z}+\frac{1}{2} \sigma\left(i_{0}\right)^{2} X(s)^{2} \frac{\partial^{2} \phi(X(s), Z(s))}{\partial x^{2}} \\
& \left.+Q(u) \psi(X(s), Z(s), \cdot)\left(i_{0}\right)-\rho \psi\left(X(s), Z(s), i_{0}\right)\right] d s .
\end{aligned}
$$

Recall that $\left(x_{0}, z_{0}\right)$ is a local minimum of $v\left(x, z, i_{0}\right)-\phi(x, z)$. Then for $0 \leq t \leq$ $\theta$, we have

$$
\begin{aligned}
v\left(X(t), Z(t), i_{0}\right) & \geq \phi(X(t), Z(t))+v\left(x_{0}, z_{0}, i\right)-\phi\left(x_{0}, z_{0}\right) \\
& =\psi\left(X(t), Z(t), i_{0}\right) .
\end{aligned}
$$

In addition, (13) implies that

$$
Q(u) \psi(X(s), Z(s), \cdot)\left(i_{0}\right) \geq Q(u) v(X(s), Z(s), \cdot)\left(i_{0}\right) .
$$

Therefore, it follows that

$$
\begin{aligned}
& E e^{-\rho \theta} v\left(X(\theta), Z(\theta), i_{0}\right)-v\left(x_{0}, z_{0}, i_{0}\right) \\
\geq \quad & E \int_{0}^{\theta} e^{-\rho s}\left[\left(\mu\left(i_{0}\right) X(s)-c u(s)\right) \frac{\partial \phi(X(s), Z(s))}{\partial x}-u(s) \frac{\partial \phi(X(s), Z(s))}{\partial z}\right. \\
& +\frac{1}{2} \sigma\left(i_{0}\right)^{2} X(s)^{2} \frac{\partial^{2} \phi(X(s), Z(s))}{\partial x^{2}}+Q(u) \psi(X(s), Z(s), \cdot)\left(i_{0}\right) \\
& \left.-\rho v\left(X(s), Z(s), i_{0}\right)\right] d s \\
\geq \quad & E \int_{0}^{\theta} e^{-\rho s}\left[\left(\mu\left(i_{0}\right) X(s)-c u(s)\right) \frac{\partial \phi(X(s), Z(s))}{\partial x}-u(s) \frac{\partial \phi(X(s), Z(s))}{\partial z}\right. \\
& +\frac{1}{2} \sigma\left(i_{0}\right)^{2} X(s)^{2} \frac{\partial^{2} \phi(X(s), Z(s))}{\partial x^{2}}+Q(u) v(X(s), Z(s), \cdot)\left(i_{0}\right) \\
& \left.-\rho v\left(X(s), Z(s), i_{0}\right)\right] d s .
\end{aligned}
$$

Combining (10) and (14), we attain

$$
0 \geq E \int_{0}^{\theta} e^{-\rho s}\left[\left(\mu\left(i_{0}\right) X(s)-c u(s)\right) \frac{\partial \phi(X(s), Z(s))}{\partial x}-u(s) \frac{\partial \phi(X(s), Z(s))}{\partial z}\right.
$$




$$
\begin{aligned}
& +\frac{1}{2} \sigma\left(i_{0}\right)^{2} X(s)^{2} \frac{\partial^{2} \phi(X(s), Z(s))}{\partial x^{2}} g(X(s), u(s))+Q(u) v(X(s), Z(s), \cdot)\left(i_{0}\right) \\
& \left.-\rho v\left(X(s), Z(s), i_{0}\right)\right] d s .
\end{aligned}
$$

Letting $\theta \rightarrow 0$, we conclude that

$$
\begin{aligned}
\max _{u \in \Gamma} & {\left[\left(\mu\left(i_{0}\right) x_{0}-c u\right) \frac{\partial \phi\left(x_{0}, z_{0}\right)}{\partial x}-u \frac{\partial v\left(x_{0}, z_{0}\right)}{\partial z}+\frac{1}{2} \sigma\left(i_{0}\right)^{2} x_{0}^{2} \frac{\partial^{2} \phi\left(x_{0}, z_{0}\right)}{\partial x^{2}}\right.} \\
& \left.+g\left(x_{0}, u\right)+Q(u) v\left(x_{0}, z_{0}, \cdot\right)\left(i_{0}\right)\right]-\rho v\left(x_{0}, z_{0}, i_{0}\right) \leq 0
\end{aligned}
$$

It implies that $v$ is a viscosity supersolution.

We need to show that $v$ is a viscosity subsolution on $\bar{\Omega}$. Assume the opposite, i.e., for $i_{0} \in \mathcal{M}$, there exist $x_{0}, z_{0} \in \bar{\Omega}$ and $\delta>0$ such that for $u \in \Gamma$,

$$
\begin{aligned}
& \left(\mu\left(i_{0}\right) x-c u\right) \frac{\partial \phi(x, z)}{\partial x}-u \frac{\partial v(x, z)}{\partial z}+\frac{1}{2} \sigma\left(i_{0}\right)^{2} x^{2} \frac{\partial^{2} \phi(x, z)}{\partial x^{2}}+g(x, u) \\
& +Q(u) v(x, z, \cdot)\left(i_{0}\right)-\rho v\left(x, z, i_{0}\right) \leq-\delta .
\end{aligned}
$$

in the neighborhood $N\left(x_{0}, z_{0}\right)$, where $\phi(x, y) \in C^{2,1}(\bar{\Omega})$ such that $v\left(x, z, i_{0}\right)-$ $\phi(x, z)$ attains its maximum at $\left(x_{0}, z_{0}\right)$ in $N\left(x_{0}, z_{0}\right)$. Without loss of generality we can assume that $v\left(x_{0}, z_{0}, i_{0}\right)-\phi\left(x_{0}, z_{0}\right)=0$. Let $u(\cdot) \in \mathcal{A}\left(x_{0}, z_{0}, i_{0}\right)$ and $\tau$ be the first jump time of the process $\alpha(\cdot)$. Let $\tau_{0} \leq \tau$ be a stopping time such that for $0 \leq s \leq \tau_{0} \leq \tau,(X(s), Z(s)) \in N\left(x_{0}, z_{0}\right)$ and $v\left(X(s), Z(s), i_{0}\right)-$ $\phi(X(s), Z(s)) \leq 0$. Then for $0 \leq \theta \leq \tau_{0}$,

$$
\begin{aligned}
J\left(x_{0}, z_{0}, i, u\right) & \leq E \int_{0}^{\theta} e^{-\rho s} g(X(s), u(s)) d s+E e^{-\rho \theta} v\left(X(\theta), Z(\theta), i_{0}\right) \\
& \leq E \int_{0}^{\theta} e^{-\rho s} g(X(s), u(s)) d s+E e^{-\rho \theta} \phi(X(\theta), Z(\theta)) .
\end{aligned}
$$

We use $(11)$, and since $\left(x_{0}, z_{0}\right)$ is a local maximum of $v\left(x, z, i_{0}\right)-\phi(x, z)$, for $0 \leq t \leq \theta$, we derive

(15) $v\left(X(t), Z(t), i_{0}\right) \leq \phi(X(t), Z(t))+v\left(x_{0}, z_{0}, i_{0}\right)-\phi\left(x_{0}, z_{0}\right) \psi\left(X(t), Z(t), i_{0}\right)$.

Similarly with (13), (15) implies that

$$
Q(u) \psi(X(s), Z(s), \cdot)\left(i_{0}\right) \leq Q(u) v(X(s), Z(s), \cdot)\left(i_{0}\right) .
$$

Using (4), (12) and (15), we have

$$
\begin{aligned}
& J\left(x_{0}, z_{0}, i, i_{0}, u\right) \\
\leq & E \int_{0}^{\theta} e^{-\rho s}\left[-\delta+\rho v\left(X(s), Z(s), i_{0}\right)\right. \\
& -\left(\mu\left(i_{0}\right) X(s)-c u\right) \frac{\partial \phi(X(s), Z(s))}{\partial x}+u \frac{\partial v(X(s), Z(s))}{\partial z} \\
& \left.-\frac{1}{2} \sigma\left(i_{0}\right)^{2} X(s)^{2} \frac{\partial^{2} \phi(X(s), Z(s))}{\partial x^{2}}-Q(u) v(X(s), Z(s), \cdot)\left(i_{0}\right)\right] d s \\
& +E e^{-\rho \theta} \phi(X(\theta), Z(\theta))
\end{aligned}
$$




$$
\begin{aligned}
\leq & E \int_{0}^{\tau} e^{-\rho s}\left[-\delta+\rho v\left(X(s), Z(s), i_{0}\right)\right. \\
& -\left(\mu\left(i_{0}\right) X(s)-c u\right) \frac{\partial \phi(X(s), Z(s))}{\partial x}+u \frac{\partial v(X(s), Z(s))}{\partial z} \\
& \left.-\frac{1}{2} \sigma\left(i_{0}\right)^{2} X(s)^{2} \frac{\partial^{2} \phi(X(s), Z(s))}{\partial x^{2}}-Q(u) \psi(X(s), Z(s), \cdot)\left(i_{0}\right)\right] d s \\
& +E e^{-\rho \theta} \psi(X(\theta), Z(\theta)) \\
\leq & -E \int_{0}^{\tau} e^{-\rho s} \delta d s+\phi\left(x_{0}, z_{0}\right) \\
\leq & -E \int_{0}^{\tau} e^{-\rho s} \delta d s+v\left(x_{0}, z_{0}, i_{0}\right) .
\end{aligned}
$$

Therefore, we have

$$
J\left(x_{0}, z_{0}, i, i_{0}, u\right) \leq-E \int_{0}^{\tau} e^{-\rho s} \delta d s+v\left(x_{0}, z_{0}, i_{0}\right) .
$$

This contradicts the fact that $\delta$ is positive, and we conclude that $v\left(x, z, i_{0}\right)$ is a viscosity subsolution.

The uniqueness of viscosity solution can be obtained in Jakobsen and Karlsen [4]. This completes the proof.

\subsection{Boundary conditions}

We consider the case when $v(\cdot, \cdot, i) \in C^{2,1}(\bar{\Omega})$ for each $i \in \mathcal{M}$. For the case of $i=1, v(x, z, 1)$ satisfies (5). Let us take $\phi(x, z)=v(x, z, 1)+\beta(N-z)$ with $\beta>0$. Then $v(x, z, 1)-\phi(x, z)$ attains its minimum at $\left(x_{0}, 0\right)$. Thus (4) is satisfied as well. Consider the combination of (4) and (5). We have

$$
\begin{aligned}
& \max _{u \in \Gamma}\left\{-c u \frac{\partial \phi\left(x_{0}, 0\right)}{\partial x}-u \frac{\partial \phi\left(x_{0}, 0\right)}{\partial z}+g\left(x_{0}, u\right)+a_{1}\left(v\left(x_{0}, 0,2\right)-v\left(x_{0}, 0,1\right)\right) u\right\} \\
= & \max _{u \in \Gamma}\left\{-c u \frac{\partial v\left(x_{0}, 0,1\right)}{\partial x}-u\left(\frac{\partial v\left(x_{0}, 0,1\right)}{\partial z}-\beta\right)+g\left(x_{0}, u\right)+a_{1}\left(v\left(x_{0}, 0,2\right)-v\left(x_{0}, 0,1\right)\right) u\right\} \\
\leq & \max _{u \in \Gamma}\left\{-c u \frac{\partial v\left(x_{0}, 0,1\right)}{\partial x}-u \frac{\partial v\left(x_{0}, 0,1\right)}{\partial z}+g\left(x_{0}, u\right)+a_{1}\left(v\left(x_{0}, 0,2\right)-v\left(x_{0}, 0,1\right)\right) u\right\} .
\end{aligned}
$$

Let $u^{*}$ be the optimal selling rate in $\Gamma$. Since $\beta>0$, to satisfy the above inequality, we have $u^{*}=0$ and the following boundary condition

$$
-c \frac{\partial v\left(x_{0}, 0,1\right)}{\partial x}-\frac{\partial v\left(x_{0}, 0,1\right)}{\partial z}+g_{0}\left(x_{0}\right)+a_{1}\left(v\left(x_{0}, 0,2\right)-v\left(x_{0}, 0,1\right)\right) \leq 0 .
$$

Similarly, if $i=2$, we have

$$
-c \frac{\partial v\left(x_{0}, 0,2\right)}{\partial x}-\frac{\partial v\left(x_{0}, 0,2\right)}{\partial z}+g_{0}\left(x_{0}\right)+a_{2}\left(v\left(x_{0}, 0,1\right)-v\left(x_{0}, 0,2\right)\right) \leq 0 .
$$

There is no constraint at $z=N$ since $u(t) \geq 0$ makes $z(t) \geq 0$ for all $t$. 


\subsection{Optimal liquidation}

In this section, we give a verification theorem for the value function along the standard way in Fleming and Rishel [1].

Theorem 3.4. For each $i \in \mathcal{M}$, let $w(\cdot, \cdot, i) \in C_{b}^{2,1}(\bar{\Omega})$ be a solution to the HJB equation (3). Then,

(a) $w(x, z, i) \geq J(x, z, i, u(\cdot))$ for all admissible $u(\cdot)$.

(b) Define the selling rate for each $i \in\{1,2\}$,

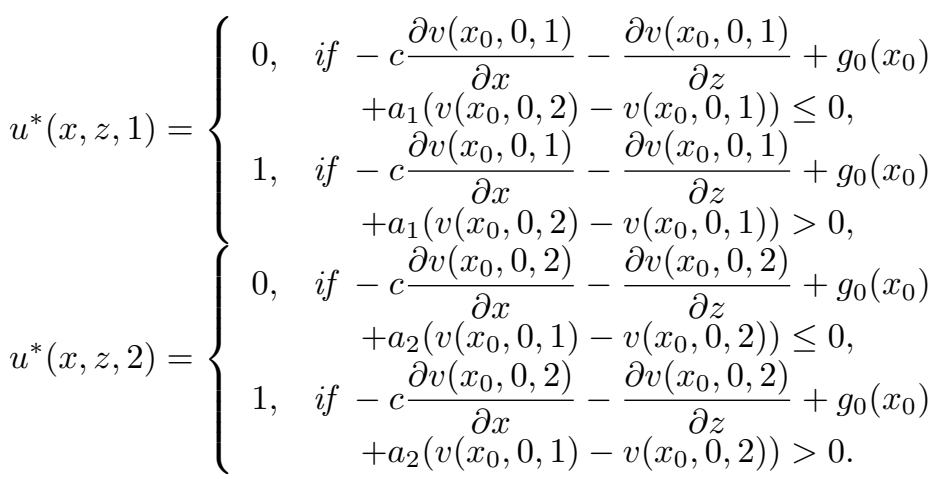

Then

$$
w(x, z, i)=v(x, z, i)=J\left(x, z, i, u^{*}(\cdot)\right) .
$$

That is, $u^{*}$ is optimal.

Proof. Using Dynkin's formula and HJB equation (3), we have

$$
E e^{-\rho T} v(X(T), Z(T), \alpha(T))-v(x, z, i) \leq-E \int_{0}^{T} e^{-\rho t} g(X(t), u(t)) d t .
$$

Sending $T \rightarrow \infty$, we have

$$
v(x, z, i) \geq E \int_{0}^{\infty} e^{-\rho t} g(X(t), u(t)) d t .
$$

The equality holds if $u=u^{*}$ given in (17). See Zhou [10].

It is very difficult task to find the closed-form solution of the system of HJB equations, even we found the optimal selling strategy. As the alternative solution, we discuss about the numerical solution of the problem by finite difference method in next section.

\section{A numerical scheme}

Let us construct an explicit finite difference scheme and show that it converges to the unique viscosity solution of the equation (3). We recall the HJB equation given by

$$
\rho v(x, z, i)=\max _{u \in \Gamma}\left[(\mu(i) x-c u) \frac{\partial v(x, z, i)}{\partial x}-u \frac{\partial v(x, z, i)}{\partial z}+\frac{1}{2} \sigma^{2}(i) x^{2} \frac{\partial^{2} v(x, z, i)}{\partial x^{2}}\right.
$$




$$
+g(x, u)+Q(u) v(x, z, \cdot)(i)]
$$

where $0 \leq z \leq N, i \in\{1,2\}$. The associated Hamiltonian is

$$
\begin{aligned}
& \mathcal{H}\left(x, z, i, v, D_{x} v, D_{z} v, D_{x}^{2} v\right) \\
= & \rho v(x, z, i)-\max _{u \in \Gamma}\left[(\mu(i) x-c u) D_{x} v(x, z, i)\right. \\
& -u D_{z} v(x, z, i)+\frac{1}{2} \sigma^{2}(i) x^{2} D_{x}^{2} v(x, z, i)+g(x, u) \\
& +Q(u) v(x, z, \cdot)(i)] .
\end{aligned}
$$

Then, we are going to approximate the solution of the equation

$$
\begin{aligned}
& \max _{u \in \Gamma}\left[(\mu(i) x-c u) \frac{\partial v(x, z, i)}{\partial x}-u \frac{\partial v(x, z, i)}{\partial z}+\frac{1}{2} \sigma^{2}(i) x^{2} \frac{\partial^{2} v(x, z, i)}{\partial x^{2}}\right. \\
& +g(x, u)+Q(u) v(x, z, \cdot)(i)]-\rho v(x, z, i)=0
\end{aligned}
$$

for $(x, z, i) \in \mathbb{R} \times[0, N] \times \mathcal{M}$. It can be shown that (19) has a unique viscosity solution $v(x, z, i)$. Let $B(\mathbb{R} \times[0, N] \times \mathcal{M})$ be the space of bounded functions $v(x, z, i)$ defined on $\mathbb{R} \times[0, N] \times \mathcal{M}$ and continuous in $(x, z)$. Let $h=\Delta x, k=\Delta z$ be the pair of step sizes between 0 and 1 for discretizing the variable $x$ and $z$. Consider the finite difference operators $\Delta_{x}, \Delta_{z}$ and $\Delta_{x}^{2}$ as

$$
\begin{aligned}
\Delta_{x} v(x, z, i) & =\frac{v(x+h, z, i)-v(x, z, i)}{h}, \\
\Delta_{z} v(x, z, i) & =\frac{v(x, z, i)-v(x, z-k, i)}{k}, \\
\Delta_{x}^{2} v(x, z, i) & =\frac{v(x+h, z, i)+v(x-h, z, i)-2 v(x, z, i)}{h^{2}} .
\end{aligned}
$$

The associate discrete version of the equation (19) is given by

$$
\begin{aligned}
& \max _{u \in \Gamma}\left[(\mu(i) x-c u) \frac{v(x+h, z, i)-v(x, z, i)}{h}-u \frac{v(x, z, i)-v(x, z-k, i)}{k}\right. \\
& +\frac{1}{2} \sigma^{2}(i) x^{2} \frac{v(x+h, z, i)+v(x-h, z, i)-2 v(x, z, i)}{h^{2}}+g(x, u) \\
& \left.+\lambda_{i}(u)(v(x, z, j)-v(x, z, i))\right]-\rho v(x, z, i)=0, \quad j \neq i .
\end{aligned}
$$

We rearrange terms to have the following

$$
\begin{aligned}
& \max _{u \in \Gamma}\left\{\frac { 1 } { \frac { \mu ( i ) x - c u } { h } + \frac { u } { k } + \frac { \sigma ( i ) ^ { 2 } x ^ { 2 } } { h ^ { 2 } } + \lambda _ { i } ( u ) } \left[\left(\frac{\mu(i) x-c u}{h}+\frac{\sigma(i)^{2} x^{2}}{2 h^{2}}\right) v(x+h, z, i)\right.\right. \\
& \left.\left.+\frac{\sigma(i)^{2} x^{2}}{2 h^{2}} v(x-h, z, i)+\frac{u}{k} v(x, z-k, i)+g(x, u)+\lambda_{i}(u) v(x, z, j)\right]\right\} \\
= & v(x, z, i),
\end{aligned}
$$

where $j \neq i$. 


\subsection{Convergence of the finite difference method}

Define a mapping $S:(0,1) \times(0,1) \times \mathcal{M} \times \mathbb{R} \times[0, N] \times \mathbb{R} \times B(\mathbb{R} \times[0, N] \times \mathcal{M}) \rightarrow$ $\mathbb{R}$ such as

$$
\begin{aligned}
& S(h, k, i, x, z, f, v) \\
= & h f-\max _{u \in \Gamma}\left\{\frac{h}{\frac{\mu(i) x-c u}{h}+\frac{u}{k}+\frac{\sigma(i)^{2} x^{2}}{h^{2}}+\lambda_{i}(u)} \times\right. \\
& {\left[\left(\frac{\mu(i) x-c u}{h}+\frac{\sigma(i)^{2} x^{2}}{2 h^{2}}\right) v(x+h, z, i)+\frac{\sigma(i)^{2} x^{2}}{2 h^{2}} v(x-h, z, i)\right.} \\
& \left.\left.+\frac{u}{k} v(x, z-k, i)+g(x, u)+\lambda_{i}(u) v(x, z, j)\right]\right\},
\end{aligned}
$$

where $j \neq i, i, j \in \mathcal{M}$. It is easy to see that (20) is equivalent to $S(h, k, i, x, z, f$, $v)=0$. Note that the coefficient of $v$ in $S$ is negative. It means that $S$ is monotone in $v$, i.e.,

$$
S\left(h, k, i, x, z, f, v_{1}\right) \leq S\left(h, k, i, x, z, f, v_{2}\right)
$$

whenever $v_{1} \geq v_{2}$.

Definition 4.1. The scheme $S$ is said to be consistent if $\lim _{\xi \rightarrow x, \zeta \rightarrow z, h \downarrow 0, k \downarrow 0, \epsilon \downarrow 0} \frac{S(h, k, i, \xi, \zeta, v(\xi, \zeta, i), v+\epsilon)}{h}=\mathcal{H}\left(x, z, i, v, D_{x} v, D_{z} v, D_{x}^{2} v\right)$.

Lemma 4.2. The scheme $S$ is consistent.

Proof. Let $\left.v(\cdot, \cdot, i) \in C^{2,1}(\bar{\Omega})\right)$ for each $i \in \mathcal{M}$. We write

$$
\begin{aligned}
& \frac{S(h, k, i, \xi, \zeta, v(\xi, \zeta, i), v)}{h} \\
= & f-\max _{u \in \Gamma}\left\{\frac{1}{\frac{\mu(i) \xi-c u}{h}+\frac{u}{k}+\frac{\sigma(i)^{2} \xi^{2}}{h^{2}}+\lambda_{i}(u)} \times\right. \\
& {\left[\left(\frac{\mu(i) \xi-c u}{h}+\frac{\sigma(i)^{2} \xi^{2}}{2 h^{2}}\right) v(\xi+h, \zeta, i)+\frac{\sigma(i)^{2} \xi^{2}}{2 h^{2}} v(\xi-h, \zeta, i)\right.} \\
& \left.\left.+\frac{u}{k} v(\xi, \zeta-k, i)+g(\xi, u)+\lambda_{i}(u) v(\xi, \zeta, j)\right]\right\} .
\end{aligned}
$$

Sending $\xi \rightarrow x, \zeta \rightarrow z, h \rightarrow 0, k \rightarrow 0$ and $\epsilon \rightarrow 0$, we can see that

$$
\frac{S(h, k, i, \xi, \zeta, v(\xi, \zeta, i), v)}{h} \rightarrow \mathcal{H}\left(x, z, i, v, D_{x} v, D_{z} v, D_{x}^{2} v\right) .
$$


Define an operator $T_{h, k}$ on $B(\mathbb{R} \times[0, N] \times \mathcal{M})$ such that

$$
\begin{aligned}
T_{h, k} v(x, z, i)= & \max _{u \in \Gamma}\left\{\frac{1}{\frac{\mu(i) \xi-c u}{h}+\frac{u}{k}+\frac{\sigma(i)^{2} \xi^{2}}{h^{2}}+\lambda_{i}(u)} \times\right. \\
& {\left[\left(\frac{\mu(i) \xi-c u}{h}+\frac{\sigma(i)^{2} \xi^{2}}{2 h^{2}}\right) v(\xi+h, \zeta, i)+\frac{\sigma(i)^{2} \xi^{2}}{2 h^{2}} v(\xi-h, \zeta, i)\right.} \\
& \left.\left.+\frac{u}{k} v(\xi, \zeta-k, i)+g(\xi, u)+\lambda_{i}(u) v(\xi, \zeta, j)\right]\right\} .
\end{aligned}
$$

Lemma 4.3. For each $h$ and $k, T_{h, k}$ is a contraction map.

Proof. To prove the lemma, we need to show that there exists $0<\beta<1$ such that

$$
\left\|T_{h, k} f-T_{h, k} g\right\| \leq \beta\|f-g\|
$$

for all $f, g \in B(\mathbb{R} \times[0, N] \times \mathcal{M})$ where $\|\cdot\|$ is the sup norm. Define $c_{h, k}$ as

$$
c_{h, k}(u)=\rho+\frac{\mu(i) \xi-c u}{h}+\frac{u}{k}+\frac{\sigma(i)^{2} \xi^{2}}{h^{2}}+\lambda_{i}(u) .
$$

Then, we have

$$
\begin{aligned}
& T_{h, k} \phi(x, z, i)-T_{h, k} \psi(x, z, i) \\
= & \max _{u \in \Gamma}\left\{\frac { 1 } { c _ { h , k } } \left[\left(\frac{\mu(i) x-c u}{h}+\frac{\sigma(i)^{2} x^{2}}{2 h^{2}}\right) \phi(x+h, z, i)+\frac{\sigma(i)^{2} x^{2}}{2 h^{2}} \phi(x-h, z, i)\right.\right. \\
& \left.\left.+\frac{u}{k} \phi(x, z-k, i)+g(x, u)+\lambda_{i}(u) \phi(x, z, j)\right]\right\} \\
& -\max _{u \in \Gamma}\left\{\frac { 1 } { c _ { h , k } } \left[\left(\frac{\mu(i) x-c u}{h}+\frac{\sigma(i)^{2} x^{2}}{2 h^{2}}\right) \psi(x+h, z, i)+\frac{\sigma(i)^{2} x^{2}}{2 h^{2}} \psi(x-h, z, i)\right.\right. \\
& \left.\left.+\frac{u}{k} \psi(x, z-k, i)+g(x, u)+\lambda_{i}(u) \psi(x, z, j)\right]\right\} .
\end{aligned}
$$

For all $x, y, i$, we have the following inequality

$$
\left|T_{h, k} \phi(x, z, i)-T_{h, k} \psi(x, z, i)\right| \leq \max _{u \in \Gamma}\left[\frac{\frac{\mu(i) x-c u}{h}+\frac{u}{k}+\frac{\sigma(i)^{2} x^{2}}{h^{2}}+\lambda_{i}(u)}{c_{h, k}(u)}\right]\|\phi-\psi\| .
$$

Moreover, we know that

$$
\frac{\frac{\mu(i) x-c u}{h}+\frac{u}{k}+\frac{\sigma(i)^{2} x^{2}}{h^{2}}+\lambda_{i}(u)}{c_{h, k}(u)}=\frac{c_{h, k}-\rho}{c_{h, k}(u)}<1
$$

and since $u \in[0,1]$, we have

$$
\max _{u \in \Gamma}\left[\frac{\frac{\mu(i) x-c u}{h}+\frac{u}{k}+\frac{\sigma(i)^{2} x^{2}}{h^{2}}+\lambda_{i}(u)}{c_{h, k}(u)}\right]<1 .
$$

Hence, we conclude

$$
\left|T_{h, k} \phi(x, z, i)-T_{h, k} \psi(x, z, i)\right|<\beta_{h, k}|| \phi-\psi \| \mid
$$

with $\beta=\beta_{h, k}<1$. 
Definition 4.4. The scheme $S$ is said to be stable if there exists a bounded solution $v_{h, k} \in B(\mathbb{R} \times[0, N] \times \mathcal{M})$ to the equation

$$
S(h, k, i, x, z, v(x, z, i), v)=0,
$$

with the bounded independent of $k$ and $h$ in $(0,1)$.

The strict contraction $T_{h, k}$ has a unique fixed point, let us denote as $v_{h, k}$, by the Banach fixed point theorem. Let $g(x, u) \leq M$. For given any function $v_{0} \in B(\mathbb{R} \times[0, N] \times \mathcal{M})$, we construct a sequence as $v_{n+1}=T_{h, k} v_{n}$ for $n \geq 0$. Then, clearly we have

$$
\lim _{n \rightarrow \infty} v_{n}=v_{h, k}
$$

In addition, we attain

$$
\begin{aligned}
v_{n+1}(x, z, i)= & \max _{u \in \Gamma}\left\{\frac { 1 } { c _ { h , k } ( u ) } \left[\left(\frac{\mu(i) x-c u}{h}+\frac{\sigma(i)^{2} x^{2}}{2 h^{2}}\right) v_{n}(x+h, z, i)\right.\right. \\
& +\frac{\sigma(i)^{2} x^{2}}{2 h^{2}} v_{n}(x-h, z, i)+\frac{u}{k} v_{n}(x, z-k, i)+g(x, u) \\
& \left.\left.+\lambda_{i}(u) v_{n}(x, z, j)\right]\right\} \\
& \leq \beta_{h, k}\left\|v_{n}\right\|+\frac{1}{c_{h, k}} M .
\end{aligned}
$$

Moreover, since $c_{h, k}(u)$ is an affine function of $u \in \Gamma$, we obtain

$$
\beta_{h, k}=\max _{u \in \Gamma} \frac{c_{h, k}(u)-\rho}{c_{h, k}(u)} \leq \frac{c_{h, k}(1)-\rho}{c_{h, k}(1)}<1
$$

for all $h, k>0$. It implies that

$$
\left\|v_{n+1}\right\| \leq \frac{c_{h, k}(1)-\rho}{c_{h, k}(1)}\left\|v_{n}\right\|+\frac{1}{c_{h, k}(1)} M .
$$

From (22), we have

$$
\left\|v_{n+1}\right\| \leq\left(\frac{c_{h, k}(1)-\rho}{c_{h, k}(1)}\right)^{n+1}\left\|v_{0}\right\|+\frac{M}{c_{h, k}(1)} \sum_{i=0}^{n}\left(\frac{c_{h, k}(1)-\rho}{c_{h, k}(1)}\right)^{i} .
$$

Let $n \rightarrow \infty$, we conclude that

$$
\left\|v_{h, k}\right\| \leq \frac{M}{c_{h, k}(1)} \cdot \frac{1}{1-\frac{c_{h, k}(1)-\rho}{c_{h, k}(1)}}=\frac{M}{\rho} .
$$

The boundedness of $v_{h, k}$ implies the stability of the scheme $S$ by definition.

Theorem 4.5. Let $v_{k, h}$ be the solution to (21). Then, as $h \rightarrow 0$ and $k \rightarrow 0$, the sequence $v_{k, h}$ converges locally uniformly on $\mathbb{R} \times[0, N]$ to the unique viscosity solution $v$ of (19). 
Proof. Define

$$
\begin{aligned}
& v^{*}(x, z, i)=\limsup _{\xi \rightarrow x, \zeta \rightarrow z, h \rightarrow 0, k \rightarrow 0} v_{h, k}(\xi, \zeta, i), \\
& v_{*}(x, z, i)=\liminf _{\xi \rightarrow x, \zeta \rightarrow z, h \rightarrow 0, k \rightarrow 0} v_{h, k}(\xi, \zeta, i) .
\end{aligned}
$$

We will show that $v^{*}$ and $v_{*}$ are sub- and supersolution of (19), respectively. Since the proof of the statement for $v_{*}$ is similar with the statement for $v^{*}$, we only prove the case for $v^{*}$. For each $i \in \mathcal{M}$, we want to show that

$$
\mathcal{H}\left(x, z, i, v^{*}\left(x_{0}, z_{0}, i\right), D_{x} \phi\left(x_{0}, z_{0}\right), D_{z} \phi\left(x_{0}, z_{0}\right), D_{x}^{2} \phi\left(x_{0}, z_{0}\right)\right) \leq 0
$$

for any test function $\phi \in C^{2,1}(\mathbb{R} \times[0, N])$ such that $\left(x_{0}, z_{0}\right)$ is a strictly local maximum of $v^{*}(x, z, i)-\phi(x, z)$. Without loss of generality, we may assume that $v^{*}\left(x_{0}, z_{0}, i\right)=\phi\left(x_{0}, z_{0}\right)$ and because of the stability of the scheme $S$, we can also assume that $\phi \geq 2 \sup _{h, k}\left\|v_{h, k}\right\|$ outside of the ball $B\left(\left(x_{0}, z_{0}\right), r\right)$ where $r>0$ is such that

$$
v^{*}(x, z, i)-\phi(x, z) \leq 0=v^{*}\left(x_{0}, z_{0}, i\right)-\phi\left(x_{0}, z_{0}\right) \text { in } B\left(\left(x_{0}, z_{0}\right), r\right) .
$$

This implies that there are sequences $h_{n}>0, k_{n}>0$, and $\left(\xi_{n}, \zeta_{n}\right) \in \mathbb{R} \times$ $[0, N]$ satisfying that we have $h_{n} \rightarrow 0, k_{n} \rightarrow 0, \xi \rightarrow x_{0}, \zeta \rightarrow z_{0}, h \rightarrow 0, k \rightarrow$ $0, v^{*}\left(\xi_{n}, \zeta_{n}, i\right) \rightarrow v^{*}\left(x_{0}, z_{0}, i\right)$ and $\left(\xi_{n}, \zeta_{n}\right)$ is a global maximum of $v_{h_{n}, k_{n}}-\phi$. Denote $\epsilon_{n}=v_{h_{n}, k_{n}}\left(\xi_{n}, \zeta_{n}, i\right)-\phi\left(\xi_{n}, \zeta_{n}\right)$. Obviously $\epsilon_{n} \rightarrow 0$ and

$$
v_{h_{n}, k_{n}}(x, z, i) \leq \phi(x, z)+\epsilon_{n} \text { for all }(x, z) \in \mathbb{R} \times[0, N] .
$$

We know that $S\left(h_{n}, k_{n}, i, \xi_{n}, \zeta_{n}, v_{h_{n}, k_{n}}\left(\xi_{n}, \zeta_{n}, i\right), v_{h_{n}, k_{n}}\right)=0$. The monotonicity of $S$ and (23) implies

$$
\begin{aligned}
& S\left(h_{n}, k_{n}, i, \xi_{n}, \zeta_{n}, \phi\left(\xi_{n}, \zeta_{n}\right)+\epsilon_{n}, \phi+\epsilon_{n}\right) \\
\leq & S\left(h_{n}, k_{n}, i, \xi_{n}, \zeta_{n}, v_{h_{n}, k_{n}}\left(\xi_{n}, \zeta_{n}, i\right), v_{h_{n}, k_{n}}^{N}\right)
\end{aligned}
$$

for large enough $N$. Therefore,

$$
\lim _{n} \frac{S\left(h_{n}, k_{n}, i, \xi_{n}, \zeta_{n}, \phi\left(\xi_{n}, \zeta_{n}\right)+\epsilon_{n}, \phi+\epsilon_{n}\right)}{h_{n}} \leq 0 .
$$

Eventually, we have

$$
\begin{aligned}
& \mathcal{H}\left(x, z, i, v^{*}\left(x_{0}, z_{0}, i\right), D_{x} \phi\left(x_{0}, z_{0}\right), D_{z} \phi\left(x_{0}, z_{0}\right), D_{x}^{2} \phi\left(x_{0}, z_{0}\right)\right) \\
= & \lim _{\xi \rightarrow x_{0}, \zeta \rightarrow z_{0}, h_{n} \downarrow 0, k_{n} \downarrow 0, \epsilon_{n} \downarrow 0} \frac{S\left(h_{n}, k_{n}, i, \xi_{n}, \zeta_{n}, \phi\left(\xi_{n}, \zeta_{n}\right)+\epsilon_{n}, \phi+\epsilon_{n}\right)}{h_{n}} \leq 0 .
\end{aligned}
$$

This implies that $v^{*}$ is a viscosity subsolution. Similarly we can obtain that $v_{*}$ is a viscosity supersolution. These give us the uniqueness of the viscosity solution and we see that $v=v^{*}=v_{*}$. In conclusion, the sequence $\left\{v_{h_{n}, k_{n}}\right\}_{h_{n}, k_{n}}$ converges locally uniformly to $v$. 

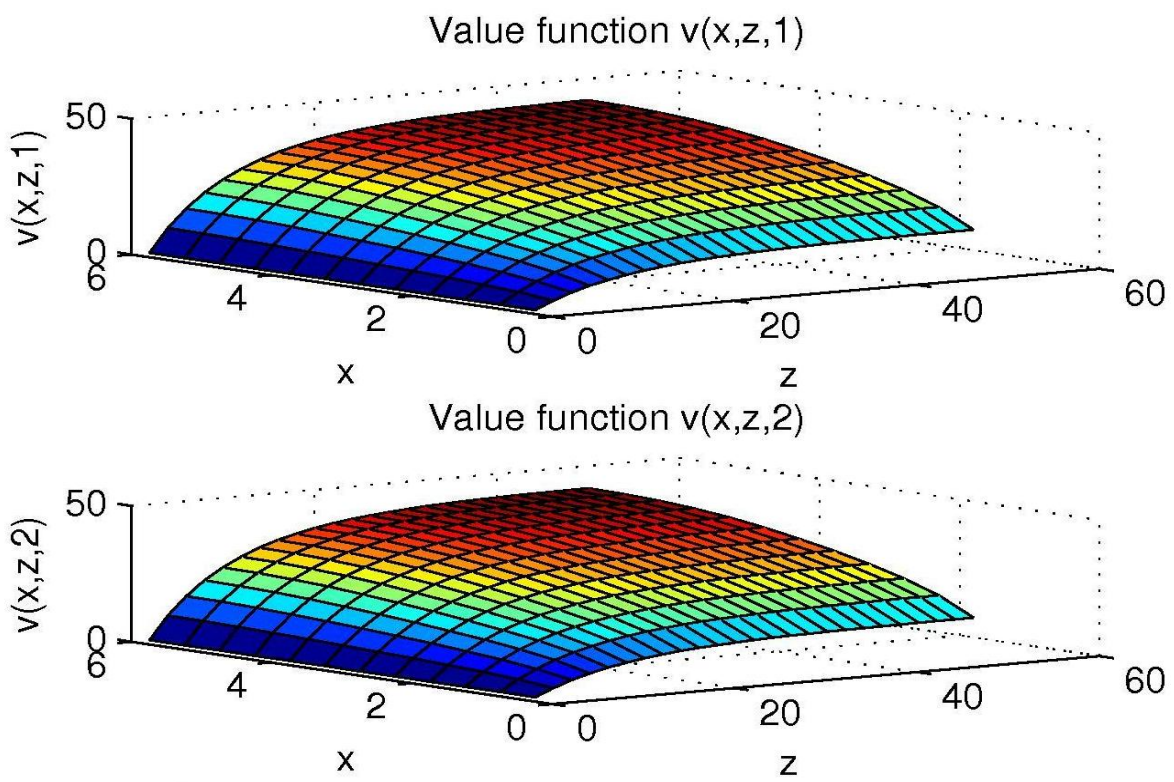

$G(x, z, 1)=x+a_{1}(v(x, z, 2)-v(x, z, 1))-c v_{x}(x, z, 1)-v_{z}(x, z, 1)$
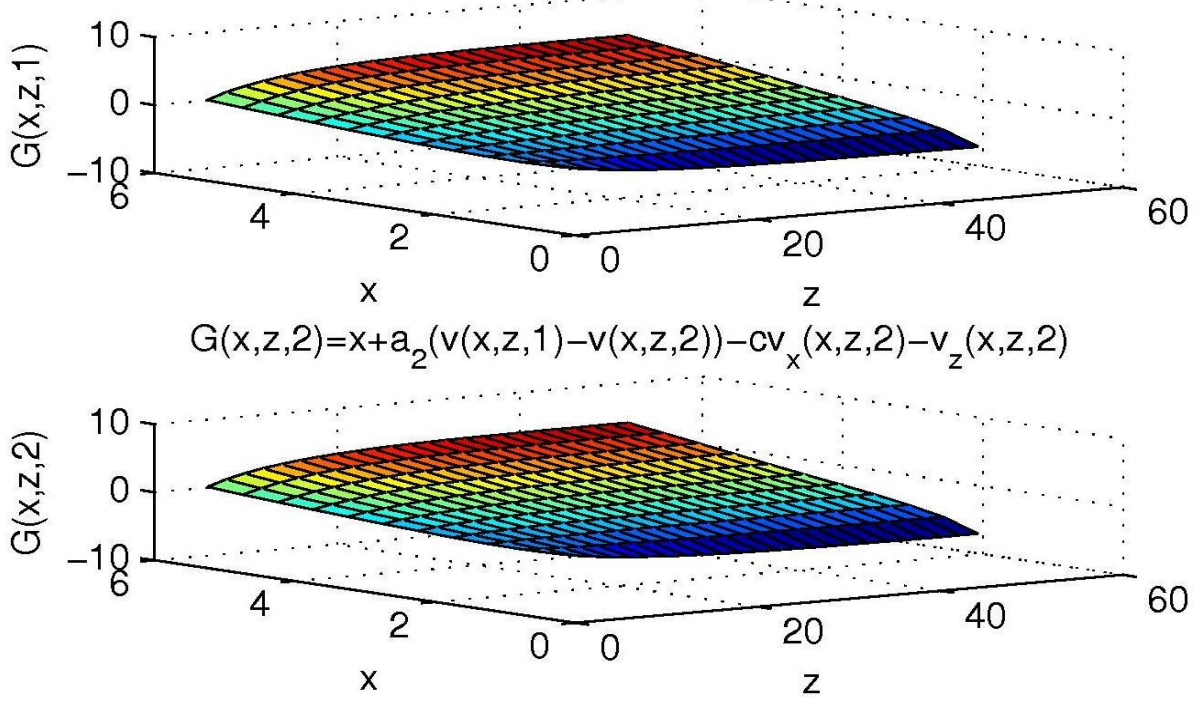

FiguRe 1. Graphs of $v(x, z, i)$ and $G(x, z, i)=x-c v_{x}(x, z, i)$ $-v_{z}(x, z, i)+a_{i}(v(x, z, j)-v(x, z, i)), i \neq j$ with $\mu(1)=$ $.3, \mu(2)=-.1, \sigma(1)=.1, \sigma(2)=.4, \lambda_{1}=4 u+1, \lambda_{2}=$ $-u+10, \rho=.1$ and $c=.3$ 


\section{A numerical example}

\subsection{Dependence of $u^{*}(x, z, i)$ on $\rho$}

In this section we execute several numerical experiments to characterize the value function and the associated optimal control policy. First, we use the introduced numerical scheme to solve the discrete version of the HJB equation. The optimal reward functions $v(x, z, 1)$ and $v(x, z, 2)$ from the numerical scheme is again used for the switching surface $G(x, z, i)=x-c v_{x}(x, z, i)-v_{z}(x, z, i)+$ $a_{i}(v(x, z, j)-v(x, z, i)), i \neq j$. The plots $v(x, z, i)$ and $G(x, z, i)$ are in Figure 1 where $\mu(1)=.3, \mu(2)=-.1, \sigma(1)=.1, \sigma(2)=.4, \lambda_{1}=4 u+1, \lambda_{2}=-u+10, \rho=$ .1 and $c=.3$ for $i=1,2$.

The optimal control $u^{*}(x, z, i)$ is determined by the curve $G(x, z, i)=0$. We can check the boundedness of the value function without the assumption $\rho>$ $\mu(1)>\mu(2)$. Through the next few subsections, we consider the dependence of the optimal selling rate $u^{*}(x, z, i)$ on some factors varied.

\subsection{Dependence of $u^{*}(x, z, i)$ on $\mu(2)$}

First, we vary the discount factor $\rho$ by $0.1,0.2,0.3$ and 0.4 . In practice, the larger discount factor, the higher discount in a future, and it stimulates selling shares. It implies that $u^{*}(x, z, i)$ should be increasing in $\rho$. In Figure 2, each curve separates the region by selling part $\left(u^{*}=1\right)$ above the curve and holding part $\left(u^{*}=0\right)$ below the curve. Figure 2 represents that the curves are decreasing in $\rho$ and means that $u^{*}(x, z, i)$ increases in $\rho$.

\subsection{Dependence of $u^{*}(x, z, i)$ on $c$}

Next, we consider the dependence of $u^{*}(x, z, i)$ on $\mu(2)$ at Figure 3. Intuitively, a larger $\mu(i)$ would encourage to hold the stock, which leads to smaller $u^{*}(x, z, i)$. In this experiments, we simply vary $\mu(2)=-0.1,0,0.1,0.2$ and observe $u^{*}(x, z, i)$. Indeed, $u^{*}(x, z, i)$ becomes smaller on $\mu(2)$.

Finally, we consider the dependence of $u^{*}(x, z, i)$ on $c$ at Figure 4. In general, the larger $c$ causes the larger selling effect on the market. It lets seller hesitate to decide selling a block of stock, and makes the $u^{*}(x, z, i)$ function smaller on c.

\section{Conclusion}

In this paper, we studied a strategy of selling a large block of a geometric Brownian motion with regime switching. In addition, a sales effect was involved into the system dynamics of the underlying stock price for reflecting negative proportion of sales. We used dynamic programming to evaluate the optimal reward function.

It was interesting how the change of a financial component, such as trends of stock and discount rate, affects to the selling rule. This paper observed the 

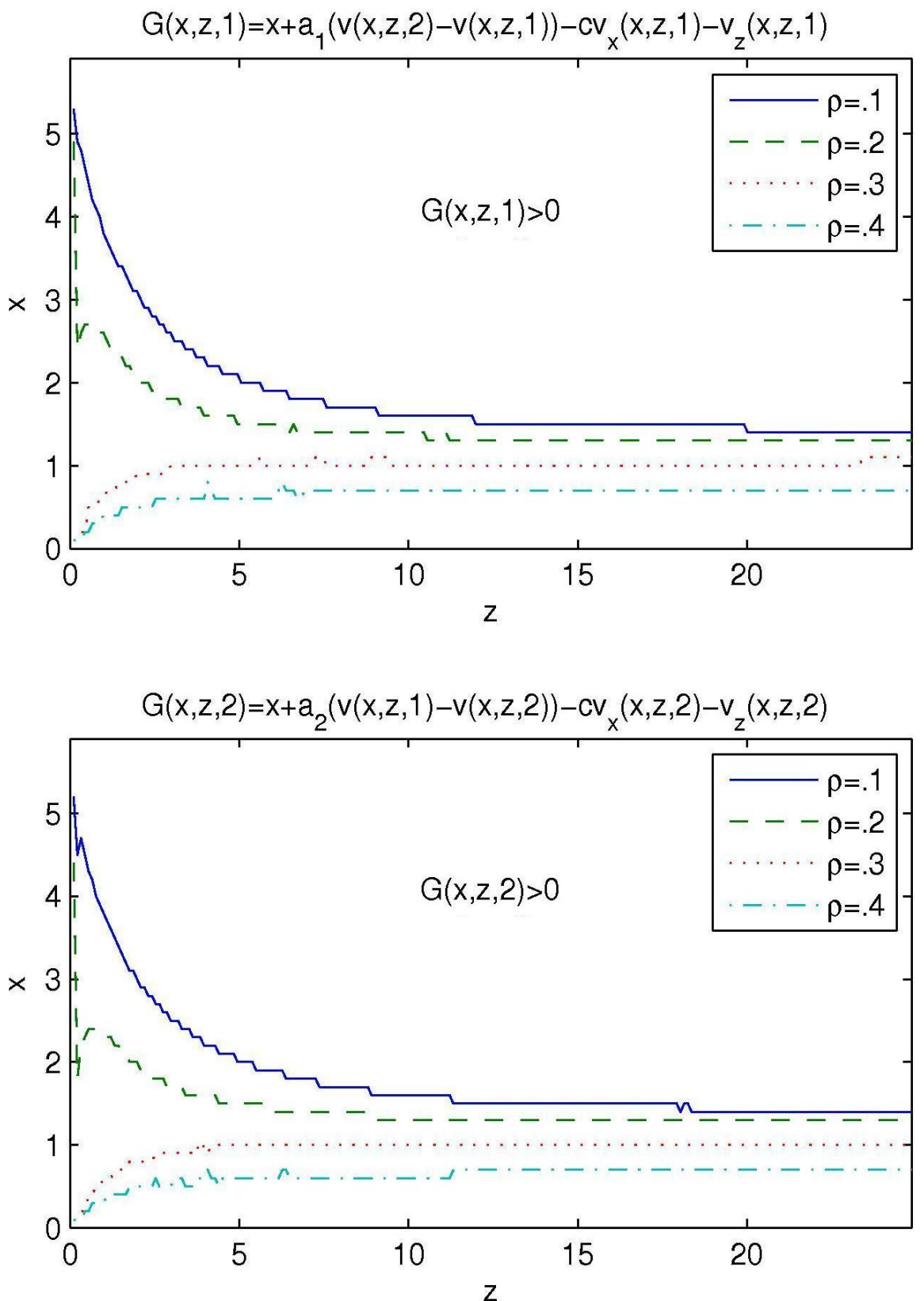

FiguRE 2. Graphs of $G(x, z, i)=0$ with varying $\rho=0.1,0.2,0.3,0.4$. 

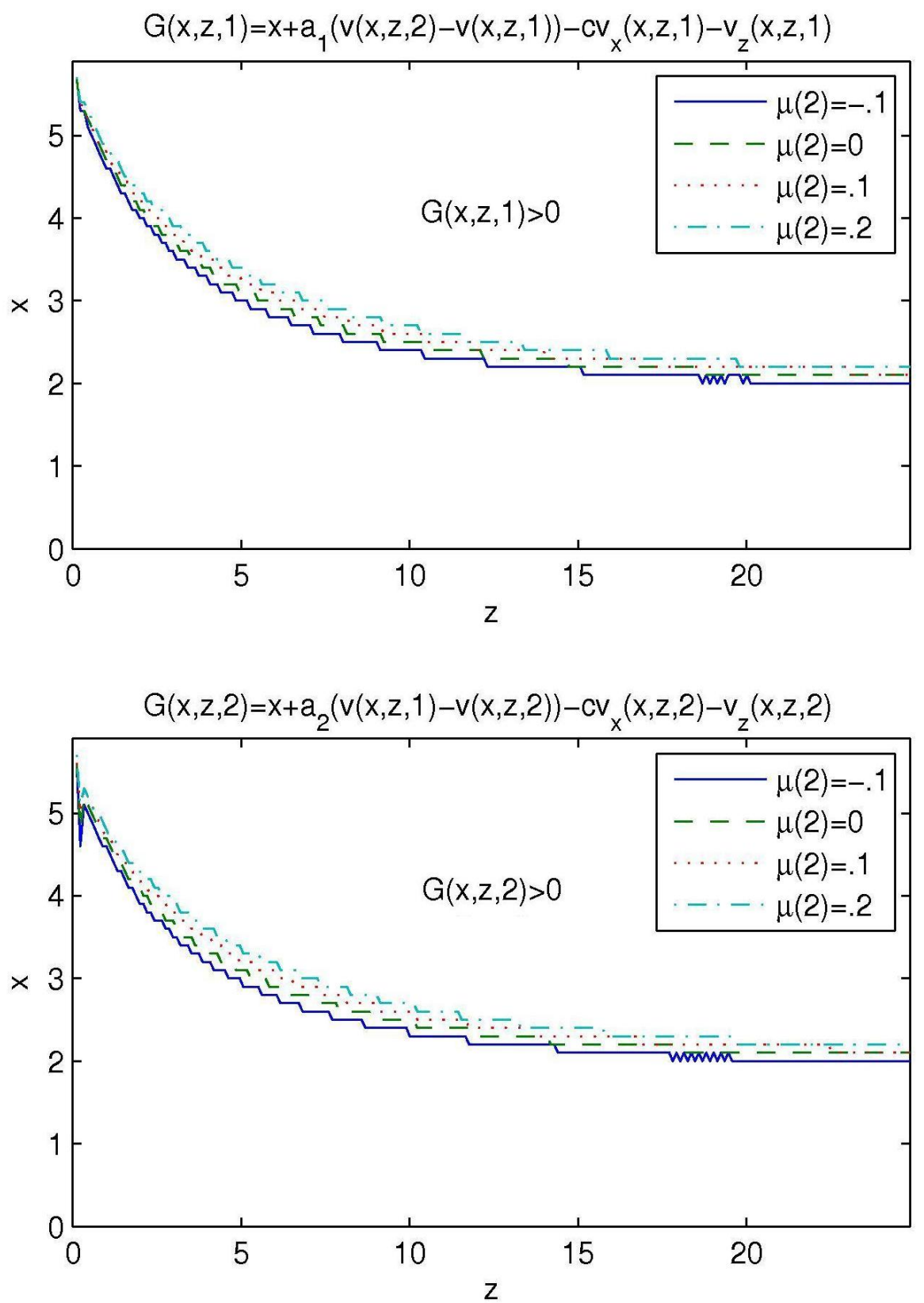

FiguRE 3. Graphs of $G(x, z, i)=0$ with varying $\mu(1)=0.3,0.4,0.5,0.6$. 

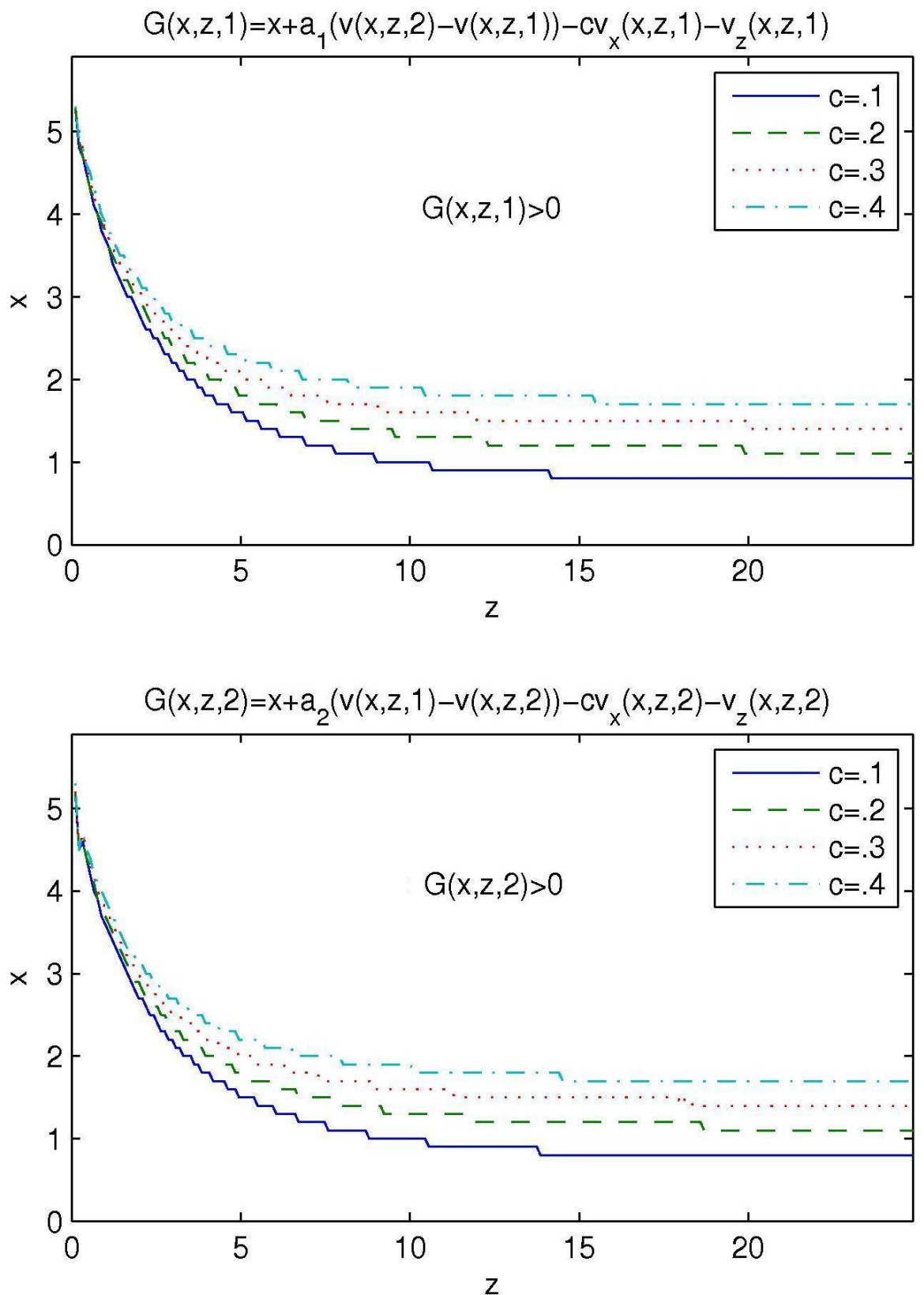

Figure 4 . Graphs of $G(x, z, i)=0$ with varying $c=0.1,0.2,0.3,0.4$. 
change effects by the simulation stated above. One may consider the same strategy about buying position.

\section{References}

[1] W. H. Fleming and R. Rishel, Deterministic and Stochastic Optimal Control, SpringerVerlag, New York, 1975.

[2] W. H. Fleming and H. M. Soner, Controlled Markov Processes and Viscosity solutions, Springer-Verlag, New York, 2005.

[3] X. Guo and Q. Zhang, Closed-form solutions for perpetual american put options with regime switching, SIAM J. Appl. Math. 64 (2004), no. 6, 2034-2049.

[4] E. R. Jakobsen and K. H. Karlsen, A "maximum principle for semicontinuous functions" applicable to integro-partial differential equations, NoDEA Nonlinear Differential Equations Appl. 13 (2006), no. 2, 137-165.

[5] B. Øksendal, Stochastic differential Equations, 6th ed., Springer-Verlag, New York, 2005.

[6] M. Pemy and Q. Zhang, Optimal stock liquidation in a regime switching model with finite time horizon, J. Math. Anal. Appl. 321 (2006), no. 2, 537-552.

[7] M. Pemy, Q. Zhang, and G. Yin, Liquidation of a large block of stock, J. Bank Finance 31 (2007), 1295-1305.

[8] Q. Zhang, Stock trading: An optimal selling rule, SIAM J. Control Optim. 40 (2001), no. $1,64-87$.

[9] Q. Zhang, G. Yin, and R. H. Liu, A near-optimal selling rule for a two-time-scale market model, Multiscale Model. Simul. 4 (2005), no. 1, 172-193.

[10] X. Y. Zhou, Verification theorems within the framework of viscosity solutions, J. Math. Anal. Appl. 177 (1993), no. 1, 208-225.

The institute of Basic Science

Korea University

SeOul 136-701, Korea

E-mail address: theater7@korea.ac.kr 